\title{
The cost of screening for colorectal cancer
}

\author{
A Walker, D K Whynes, J O Chamberlain, J D Hardcastle
}

\begin{abstract}
Study objective-The aim was to make projections of the likely costs and yield resulting from the implementation of a faecal occult blood screening programme for colorectal cancer.

Design-Cost and clinical data were derived from the MRC colorectal screening trial currently in progress in Nottingham, UK.

Setting-The above data were used as the basis for modelling the likely implications were the trial to be reproduced as a screening programme within a "typical" family practitioner committee area.

Main results-For an average family practitioner committee area with a target population of 75000 subjects aged 50-74 years, the initial screening round might be expected to detect 85 cancers at a total cost of approximately $£ 250000$. This represents a cost per cancer detected of $£ 2700$ and a cost per person screened of approximately $\mathfrak{t 5}$. For subsequent screening rounds, total costs might be expected to fall although average costs are likely to remain approximately constant.
\end{abstract}

Conclusions-The model is successful in generating "order of magnitude" estimates for the costs of implementation of a screening programme for colorectal cancer. As benefit estimates are not yet available, however, no cost-effectiveness analysis can be undertaken at this stage. In general, sensitivity analyses reveal that programme costs are more sensitive to changes in clinical variables, especially detection and compliance rates, than they are to variations in the costs of resource inputs. A screening programme with a more elaborate protocol than that currently employed in the Nottingham trial will entail considerable cost increases.

\section{University of Nottingham, University Park, \\ Nottingham NG7 \\ 2RD, United Kingdom \\ Department of \\ Surgery \\ A Walker \\ J D Hardcastle \\ Department of \\ Economics \\ D K Whynes \\ Institute of Cancer \\ Research, London \\ SW7 \\ J O Chamberlain}

Correspondence to: Dr Whynes detected visibly when the cancer is at an early stage, chemical methods achieve a far higher degree of success. The "Haemoccult" self completion test is one example of chemical detection and is being employed in a randomised controlled trial of faecal occult blood testing for colorectal cancer in Nottingham. ${ }^{3}$ To date, the trial has recruited more than 100000 persons aged 50-74 years and the results suggest that such occult blood tests may well prove to be a clinically effective tool for a programme of mass population screening, allowing colorectal adenomas and carcinomas to be detected at an early stage prior to the presentation of symptoms.

This paper uses clinical and economic data derived from the Nottingham trial to make projections of the likely health care costs and yield resulting from the implementation of such a programme. It will be appreciated that, at the present stage of the research, such a costing exercise can only be preliminary, contenting itself with orders of magnitude. Many of the relevant clinical variables are themselves not known with certainty; indeed, the principal function of the Nottingham trial, and those being conducted in parallel in other countries, is to estimate the magnitude of these variables. The organisational structure of the UK health service, moreover, is currently in a state of flux, and the financial responsibilities for the various elements involved in screening programmes have yet to be definitively allocated. For these reasons we have subjected our cost estimates to sensitivity analyses, to indicate how our results might be expected to vary with changing assumptions about the size of the principal variables.

\section{Methods}

THE NOTTINGHAM SCREENING TRIAL

Asymptomatic patients entering the Nottingham trial are identified directly from the records of local general practitioners; the criterion for inclusion is age (determined by incidence rates), subject to the absence of known serious bowel disease. Patients are allocated at random into a study or a control group $(50 \%$ of the population in each), and those in the former are sent a Haemoccult test for completion. Patients are invited to complete the test at home, by taking two pea size stool samples per day on three consecutive days, and each sample is placed on a separate sheet of chemically impregnated paper in a cardboard envelope. The completed tests are returned to, and analysed by, the Department of Surgery at the Queen's Medical Centre (QMC), Nottingham, the addition of a reagent to the samples by the analyst providing a chemical 
indication of the possibility of the presence of occult blood. In common with all such faecal occult blood tests, Haemoccult is neither $100 \%$ sensitive nor $100 \%$ specific. A positive test result might indicate not only a carcinoma but also any one of range of bowel abnormalities, or even a false positive result caused by diet or a normal level of bleeding. Occult blood may also be passed by a subject with no recognised bowel abnormality. All positive results are therefore further investigated either by colonoscopy or by double contrast barium enema $x$ ray, and those patients with detected abnormalities are referred for the appropriate treatment. Those patients complying are offered rescreening every alternate year (subject to the trial's age limits).

The main study of colorectal screening at the QMC has been in progress since October 1984 and has received a total funding in excess of $£ 600000$ (current prices). This total budget has accommodated a wide variety of specific expenditures-staff salaries, tests, equipment, stationery, and so forth-although the costs of clinical investigation and treatment are borne, where necessary, by the hospital, ie, the National Health Service (NHS). In that the QMC is a large teaching hospital and that the trial constitutes an experimental research programme, the trial costings cannot really be taken as typical of those to be expected within a mass screening programme. Accordingly, we use the QMC clinical and cost data as a basis for modelling the likely implications were the trial to be reproduced as a screening programme within an "average" family practitioner committee area, with a population of 75000 persons aged $50-74$ years. All cost figures are expressed in $1989 / 90$ prices.

\section{A MODEL OF A SCREENING PROGRAMME}

The viewpoint of the analysis adopted is that of the NHS screening programme alone. This means that the following relevant costs are excluded:

(1) Private costs-at present we have few data on this aspect, although provisional evidence suggests a private cost of clinic attendance of the order of $£ 5$ per subject. Psychological costs, moreover, may also be significant. ${ }^{4}$

(2) Differences in the costs of treatment between those cancers presenting symptomatically and those detected by screening-average costs may be lower in the screen detected group but biases such as overdiagnosis of slow growing cancers mean that the impact on total costs is uncertain.

(3) Differences in the costs of presentation between those cancers presenting symptomatically and those detected by screening-again, we have no information on this at present.

All of the above considerations are the subject of ongoing research at Nottingham.

The principal potential benefit identified but not measured is that resulting from the detection and removal of ademonas at a benign stage. It is widely accepted that some adenomas will become malignant but uncertainty in the clinical literature as to precisely how many cancers are thus prevented has deterred us from attempting to solve this problem here. The net effect of these exclusions is predicted to tilt the balance of the analysis against screening from an NHS viewpoint; potential cost savings could exist at diagnostic and treatment phases, as well as higher benefits. The limitations on the mortality and survival evidence available to us dictates that an intermediate outcome measure be used. All results are expressed in terms of an average figure: cost per cancer detected. This is unsatisfactory in that it does not allow comparisons with alternative use of resources, but at this stage of the trial we see no alternative.

The translation of the experience of screening under QMC trial conditions to a more general setting requires us to make a number of assumptions. First, it would seem likely that any mass screening programme would necessarily make use of family practitioner committee records as source material. Our experience, and that of others, suggests a degree of inaccuracy in such data (possibly up to $20 \%$ ). ${ }^{5}$ This could represent a serious problem because it implies that a proportion of distributed faecal occult blood tests would not therefore reach their intended destinations, let alone be completed or returned (for this reason, the trial itself makes use of general practice records). However, it is to be hoped that this situation will improve over time, as more use is made of these records, and for the present we shall assume that the family practitioner committee records are completely accurate and merely require regular updating.

The Nottingham trial is administered by two whole time equivalent clerical officers. These officers have a variety of additional duties which would not be relevant outside the context of the trial and we envisage that one clerical officer and two clerical assistants (at a total cost of $£ 18909$ per annum) would be sufficient to handle the necessary administration in a more general setting, assuming the appropriate computer software were to be available. In addition, a consultant surgeon acts as overseer to the entire screening project, and would presumably continue to do so in the general setting. This input amounts to one session per week and is accordingly costed at one tenth of the cost of employing the consultant ( $£ 38400$ per annum).

Again, we initially presume that each FPC will be able to provide furnished office accommodation at zero opportunity cost, and free access to the existing mainframe computer. There would, however, exist an additional requirement for a local computing budget, covering, for example, the purchase of terminals, a printer, disks and tapes, and lines to the mainframe.

We assume, as in the trial, that a screening programme will be voluntary rather than obligatory. The average compliance in the trial since its inception is around $53^{\circ}$ o although, since the beginning of $1987, \mathrm{a} 62.5^{\circ}{ }_{0}$ response has been achieved. A consistent rise in response rates suggests a real effect, and not an arbitrary product of the order in which the population has been recruited. Publicity surrounding the introduction of a local screening programme might be expected to enhance participation. For this paper, however, we have chosen to use an intermediate figure for the three day testing of $57 \cdot 8^{\circ}{ }_{0}$, as cited in a recent trial report. ${ }^{6}$ This implies, in a population of 75000 , an expected return of 43350 tests for 
processing. At present, tests are being developed by a State Registered Nurse, and we continue with this as an assumption. Experience suggests that a test processing rate of 60 tests per hour is feasible, and development is accordingly costed on the basis of employing the nurse for the necessary length of time (total annual cost of state registered nurse $=£ 9132$ ). The current cost of a three day Haemoccult test is $£ 1 \cdot 13$, including reagent (based on the purchase price of one pack of 50 tests). Each test costs $£ 0.41$ to send (including postage, stationery, and instruction leaflet). Returned unused tests are assumed to be discarded rather than reused.

Trial data indicate that $1.29 \%$ of completed tests have positive results under three day testing. ${ }^{6}$ In our model, 559 persons would therefore require a clinical investigation to be followed by a diagnostic investigation. Clinics will presumably be held at the district's general hospital; following Nottingham practice, 12 patients would be seen in one session involving both a consultant and a nurse. At the clinic a preliminary examination is made, including a rectal investigation by a rigid sigmoidoscope, and clinics have been costed on the basis of labour costs alone. The further investigation of patients with positive test results will entail colonoscopic or barium enema $x$ ray procedures. In Nottingham, the overwhelming majority of trial patients are investigated by colonoscopy, $75 \%$ of the total as day cases and $20 \%$ as inpatients. The remaining $5 \%$ are investigated by the barium enema $x$ ray method. The costs of these three modes of investigation have been estimated at $£ 107 \cdot 0$ and $£ 140 \cdot 0$, and $£ 46 \cdot 8$, respectively. ${ }^{7}$

Finally, all costings are estimated on the basis of the assumption of a two year screening round. This follows the Nottingham trial protocol and is, therefore, the only screening interval for which data are available.

\section{Results}

The overall accounts for our model appear in table I. These are presented as recurrent costs, although some element of fixed "start up" costs must also be anticipated. The computing equipment necessary to operate the screening system would entail an expenditure of the order of $£ 25000$. Administrative staff would need to be in post for perhaps six months before the programme became operationalised, at a cost of some $£ 10000$, and the nurse responsible for test development would require a short period of

Table I Recurrent costs for a screening round in a population of 75000 persons ( $\left.£^{\prime} 000\right)$.

\begin{tabular}{|c|c|c|c|}
\hline & Cost & Subtotals & ${ }^{\circ}$ Of total \\
\hline $\begin{array}{l}\text { Overhead staff costs } \\
\text { Administration } \\
\text { Consultant }\end{array}$ & $\begin{array}{r}37 \cdot 818 \\
7 \cdot 680\end{array}$ & $45 \cdot 498$ & $19 \cdot 7$ \\
\hline $\begin{array}{l}\text { Faecal occult blood tests } \\
\text { Cost of tests } \\
\text { Cost of sending tests } \\
\text { Cost of test development }\end{array}$ & $\begin{array}{r}84 \cdot 750 \\
30 \cdot 750 \\
3 \cdot 665\end{array}$ & $119 \cdot 165$ & $51 \cdot 6$ \\
\hline $\begin{array}{l}\text { Investigations } \\
\text { Initial clinic } \\
419 \text { day case colonoscopies } \\
112 \text { in patient colonoscopies } \\
28 \text { barium enema investigations }\end{array}$ & $\begin{array}{r}4 \cdot 516 \\
44 \cdot 833 \\
15 \cdot 680 \\
1 \cdot 309\end{array}$ & $66 \cdot 338$ & $28 \cdot 7$ \\
\hline Total cost & & $231 \cdot 001$ & $100 \cdot 0$ \\
\hline
\end{tabular}

training (costing, perhaps $£ 2000$ ). Fixed costs such as hospital overheads are ignored on the assumption that they would be incurred irrespective of the existence of the screening programme; computer software development costs would presumably be incurred at a national level and would not be borne by individual areas.

As may be seen from table I, the total recurrent costs of a screening round amount to approximately one quarter of a million pounds. The Nottingham trial to date has yielded a detection rate of cancers of 1.97 per 1000 persons screened on three day testing. ${ }^{6}$ In our model population, this translates to 85 cancers and the cost per cancer detected in the initial screening round is therefore $£ 2705$. The cost per test distributed is $£ 3.08$, and the cost per person screened is $£ 5.33$, under the asssumed compliance rate.

As has been made clear, our model is a simulation excercise based upon trial results and rests upon several key assumptions. It is accordingly important to subject the results to a sensitivity analysis, to highlight the importance, or otherwise, of such assumptions. For example: (1) It might be anticipated that, were faecal occult blood screening to be adopted at a national level, test costs could fall owing to economies of scale on the part of the manufacturer. A $10 \%$ reduction in the unit purchase cost of tests would, when applied to the model, result in a $3.6 \%$ fall in costs (per cancer detected, per person screened and per test administered).

(2) As the postal system is the medium of test distribution, test distribution costs would appear, intuitively, significant as a costing element. In fact, a rise of $10 \%$ in postal costs would result only in a $1.3 \%$ rise in costs per cancer detected.

(3) Were the costs of investigations to rise by $10 \%$, costs per cancer detected would increase by $2 \cdot 7 \%$.

(4) Treating all colonoscopy patients as day cases, rather than as a mix of day cases and more expensive inpatient cases, would produce a cost saving of $1.6 \%$.

(5) Administrative staff cost rises of $10 \%$ would add $1.6 \%$ to the cost per cancer detected.

(6) The scale effect based on the size of the programme operates in the predictable fashion, ie, a larger programme generates increasing total costs but falling average costs. A $10 \%$ increase in the target population, from 75000 to 82500 , raises total costs by $8.0 \%$ and lowers the cost per cancer detected and the cost per person screened by $1.8 \%$.

As can be seen, all of the above effects are relatively minor, ie, our cost estimates are only marginally influenced by departures from the basic model assumptions. By contrast, however, changes in certain clinical variables are of greater significance. Suppose, for example, the Haemoccult test is eventually found to have a lower positive predictive value than the data currently suggest, ie, the detection rate is actually $10 \%$ lower for the given positive rate. Proportionately fewer cancers will therefore be indentified and this has the effect of raising costs per cancer detected by $11 \cdot 1 \%$. As the total operating costs of the screening programme do not vary with respect to the detection rate, the cost 
per cancer is particularly sensitive to changes in the latter variable (by contrast, costs per person screened and per test sent remain unchanged). A detection rate $20 \%$ lower than that presumed, for example, generates a $£ 676(25.0 \%)$ cost increase. In fact, given the model data, the effect is approximately proportional, ie, halving the detection rate increases costs per cancer by around $105 \%$, and doubling the detection rate halves the cost. When estimating the full expense of a projected screening programme, therefore, accurate knowledge of the likely detection rate is at a premium.

Finally, table II presents the consequences of an optimistic projection. Here is it assumed that (1) with progress in medical technology, the faecal occult blood test can be made to yield a higher positive predictive value (a $10 \%$ lower positive rate for the given detection rate), and (2) as a result of a higher public profile being given to a general programme of population screening, compliance can be increased (by 10\%). The table displays the proportionate cost changes for the variation in each assumption, and also the combined effect. Under this projection, therefore, there will be a cost saving of approximately $£ 240$ per person screened and per cancer detected. By itself, the perhaps implausible assumption of a compliance rate of $100 \%$ would reduce the cost per cancer detected by $29 \cdot 4 \%$, to $£ 1910$.

\section{Discussion}

The results presented thus far have assumed a mass screening programme consistent with the Nottingham trial protocol. However, alternative screening scenarios can be envisaged, necessitating changes in the model's assumptions. Doubts over the accuracy of family practitioner committee data were mentioned earlier, for example. It is conceivable that in order to operate the screening programme, the family practitioner

Table II Sensitivity analyses for compliance and test sensitivity: change in costs from base projection $(\%)$.

\begin{tabular}{llll}
\hline & $\begin{array}{l}\text { Per cancer } \\
\text { detected }\end{array}$ & $\begin{array}{l}\text { Per test } \\
\text { sent }\end{array}$ & $\begin{array}{l}\text { Per person } \\
\text { screened }\end{array}$ \\
\hline 10\% lower positive rate for given & -2.9 & -2.9 & $-2 \cdot 9$ \\
detection rate & -2.9 & -2.9 & $-2 \cdot 3$ \\
$10 \%$ increased compliance & -6.3 & $3 \cdot 0$ & -6.3 \\
Combined effect & $-9 \cdot 2$ & $0 \cdot 1$ & -9.2 \\
\hline
\end{tabular}

Table III Comparison of 6 day with 3 day Haemoccult testing.

\begin{tabular}{lcc}
\hline & 3 Day & 6 Day \\
\hline Cost of test $(£)$ & 1.13 & 2.26 \\
Cost of sending test $(£)$ & 0.41 & 0.6 \\
Rate of developing test & 60 & 30 \\
$\quad$ (number per hour) & 57.8 & 53.8 \\
Compliance rate $(\%)$ & 1.29 & 1.69 \\
Positive rate $(\%)$ & 1.97 & 2.56 \\
Cancer detection rate & $\quad$ per 1000 accepters) & 3366 \\
Cost per cancer detected $(£)$ & 2705 & 4.66 \\
Cost per member of target & & 8.62 \\
$\begin{array}{l}\text { population }(£) \\
\text { Cost per person screened }\end{array}$ & 5.33 & \\
\hline
\end{tabular}

Table IV Costs over three screening rounds.

\begin{tabular}{lccc}
\hline & Round 1 & Round 2 & Round 3 \\
\hline Number screened & 75000 & 43350 & 33293 \\
Compliance rate $(\%)$ & 57.8 & 76.8 & 84.2 \\
Positive rate $(\%)$ & 1.29 & 1.20 & 0.47 \\
Detection rate (per 1000 screened) & 1.97 & 1.56 & 1.65 \\
Cost per cancer detected $(£)$ & 2705 & 3128 & 2481 \\
Cost per test sent $(£)$ & 3.08 & 3.78 & 3.45 \\
Cost per person screened $(£)$ & 5.33 & 4.88 & 4.09 \\
\hline
\end{tabular}

committee register (which we earlier assumed was definitive) would have to be supplemented by additional data collected from alternative sources, such as the electoral register or general practice records. Such data collection will entail the additional expenditure of administrative staff time. Compliance, moreover, will be lower than anticipated, owing to the misdirection of a proportion of the tests sent out from the screening unit. This effect can be simulated in the model by (a) increasing administrative staff costs by $50 \%$, to cope with an increased work effort in identifying screening targets, and (b) reducing compliance by $15 \%$, to allow for test misdirection. Under these assumptions, the cost per cancer detected and cost per person screened rise by $21.9 \%$, to $£ 3298$ and $£ 6.49$ respectively. The cost per test rises by $3.6 \%$, to $£ 3 \cdot 19$. The compliance fall alone accounts for $60 \%$ of the combined effect.

As part of the Nottingham trial a comparison has been made between the results of six day and three day Haemoccult testing, on the basis of the expected higher quality of the clinical results from a longer faecal occult blood test period. Were such six day testing to be envisaged within the mass screening programme, the model would require revised assumptions, derived from the trial data. ${ }^{6}$ Table III displays the model assumptions under the three day and six day scenarios, and identifies the cost consequences. As may be inferred, the six day scenario is successful in detecting more cancers, although it does so at the expense of lower compliance and higher costs associated with the test (basic test cost, distribution and development). Moreover, the higher positive rate necessitates more follow up investigations. Overall, the six day scenario generates consistently higher cost figures; increases with respect to the three day scenario are $24.4^{\circ}$ o (per cancer detected), $50 \cdot 1 \%$ (per member of the target population), and $61.7 \%$ (per person actually screened). In comparison with the three day scenario, the incremental yield and cost of six day testing is 18 cancers and $£ 116719$, giving a cost per incremental cancer detected of $£ 6484$.

The figures presented thus far relate to the initial screening round only. As rescreening in the Nottingham trial has progressed, however, data for yields in subsequent rounds have become available, although the number of subjects in later rounds is very much smaller. In the general screening context we shall presume that only those accepting screening initially are to be offered rescreening in the following periods. Accordingly, the necessary overhead staff input can be reduced (administrative staff costs down one third and a halving in the number of consultant administrative sessions required). Table IV presents the model input data and costs for the initial and subsequent screening rounds. As may be seen, the cost per cancer detected fluctuates within approximately $13^{\circ}{ }_{0}$ about a mean of $£ 2771$, although the cost per person screened declines across the three screening rounds.

A debate is currently taking place in the clinical literature relating to the most appropriate form of investigation following positive faecal occult blood test results. ${ }^{8}$ Our model follows the trial 
protocol in accepting that colonoscopy will be the principal initial investigation, although other researchers have made claims for the initial use of radiology, both on the grounds of relative predictive accuracy and comparative cost..$^{9-11}$ Moreover, it is conceivable that, in the general screening setting, access to a fully equipped endoscopy suite will not be available. Were the roles of radiology and endoscopy to be reversed in our model (ie, $95 \%$ of patients were to receive radiological examinations and 5\% colonoscopic), the cost per cancer detected and cost per person screened would fall by $14.6 \%$ to $£ 2309$ and $£ 4.55$, respectively. There exists, of course, the presumption that there is no corresponding fall in the cancer detection rate as the result of a change in procedure; this assumption is consistent with the findings of some studies ${ }^{7}$ but not with those of others. ${ }^{10}$ Were radiology to fail to detect 12 or more cancers than colonoscopy in the initial screening round then the investigation method specified in the trial protocol would prove superior in cost per cancer detected terms.

\section{CONCLUSIONS}

A cost analysis conducted on the basis of a clinical trial can only hope to produce "order of magnitude" figures. We have estimated that a mass screening programme using the Haemoccult test in an "average" family practitioner committee area would cost approximately $£ 230000$ (recurrent costs) for the intitial screening round. The cost for the detection of each cancer would be $£ 2700$, plus or minus $20 \%$, depending upon the screening scenario envisaged; the cost per test distributed would be in a similar range, about $£ 3$. Generally speaking, the costs of a screening programme which conforms to the Nottingham protocol are more sensitive to changes in clinical variables, especially detection and compliance rates, than to corresponding changes in the costs of resource inputs. This having been said, radical departures from the protocol, involving, for example, six day testing or a different $\mathrm{mix}$ of investigations, do entail substantial cost effects. Whether or not such results indicate that mass colorectal screening is cost-effective depends upon the evaluation of benefits accruing to early cancer detection, and this evaluation represents the next stage of our research.

The authors gratefully acknowledge the financial support of the Medical Research Council, assistance from Chris Mangham and Neil Barker, and helpful comments from the anonymous referee.

1 Office of Population Censuses and Surveys. Cancer statistics-regestrations, London: HMSO, 1988.

2 Stower MJ, Hardcastle JD. The results of 1115 patients with colorectal cancer treated over an 8-year period in a single hospital. Eur f Surg Oncol 1985; 11: 119-23.

3 Hardcastle JD, Chamberlain J, Sheffield J, et al. Randomised controlled trial of faecal occult blood screening for colorectal cancer: results for the first 107,344 pcreening for colorectal cancer: result

4 Marteau TM. Psychological costs of screening. BMF 1989; 299: 527.

5 Stilman A. Age-sex registers as a screening tool for general practice: size of the wrong address problem. $B M \mathcal{F}^{\prime} 1984$; 289: 415-6.

6 Thomas WM, Pye G, Mangham C, Hardcastle JD. A trial of 3-day $v$ 6-day Haemorcult screening for colorectal neoplasia (abstract). Gut 1989; 30: A734.

7 Walker A, Whynes DK, Chamberlain JO, Hardcastle JD. The hospital cost of diagnostic procedures for colorectal cancer. $\mathcal{F}$ Clin Epidemiol (in press).

8 England WL, Halls JJ, Hunt VB. Strategies for screening for colorectal cancer. Med Decis Making 1989; 9: 3-13.

9 Feczko PJ, Halpert RD. Reassessing the role of radiology in Feczko PJ, Halpert RD. Reassessing the role of radiolo
Haemoccult screening. $A f R$ 1986; 146: 687-701.

Haemoccult screening. AFR 1986; 146: 687-701.
10 Ott DJ, Gelfand DW, Yu MC, Munitz A. Colonoscopy and Ott DJ, Gelfand DW, Yu MC, Munitz A. Colonoscopy and
the barium enema: a radiologic viewpoint. South Med $\mathcal{f}$ 1985; 78: 1033-5.

11 Reiertsen O, Bakka A, Tronnes S, Gauperaa T. Routine double-contrast barium enema and fibreoptic colonoscopy in the diagnosis of colorectal cancer. Acta Chir Scand 1988; 154: $53-5$. 